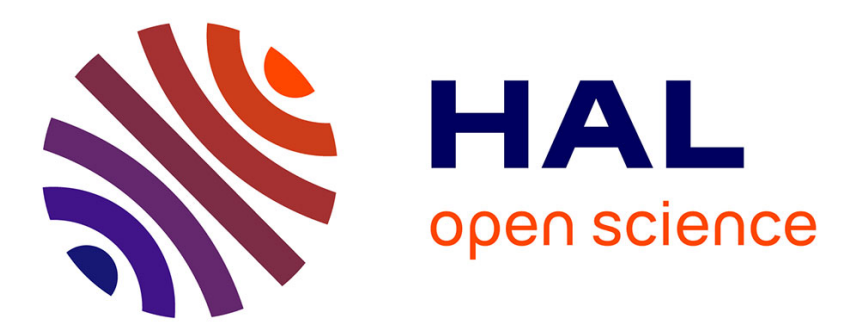

\title{
An Efficient Multi-Group Key Management Protocol for Internet of Things
}

\author{
Mohamed Ali Kandi, Hicham Lakhlef, Abdelmadjid Bouabdallah, Yacine \\ Challal
}

\section{- To cite this version:}

Mohamed Ali Kandi, Hicham Lakhlef, Abdelmadjid Bouabdallah, Yacine Challal. An Efficient MultiGroup Key Management Protocol for Internet of Things. 26th International Conference on Software, Telecommunications and Computer Networks (SoftCom 2018), Sep 2018, Split, Croatia. pp.1-6, 10.23919/softcom.2018.8555857 . hal-03281297

\section{HAL Id: hal-03281297 https://hal.science/hal-03281297}

Submitted on 8 Jul 2021

HAL is a multi-disciplinary open access archive for the deposit and dissemination of scientific research documents, whether they are published or not. The documents may come from teaching and research institutions in France or abroad, or from public or private research centers.
L'archive ouverte pluridisciplinaire $\mathbf{H A L}$, est destinée au dépôt et à la diffusion de documents scientifiques de niveau recherche, publiés ou non, émanant des établissements d'enseignement et de recherche français ou étrangers, des laboratoires publics ou privés. 


\title{
An Efficient Multi-Group Key Management Protocol for Internet of Things
}

\author{
Mohamed Ali Kandi ${ }^{1}$, Hicham Lakhlef ${ }^{1}$, Abdelmadjid Bouabdallah ${ }^{1}$ and Yacine Challal ${ }^{2}$ \\ ${ }^{1}$ Heudiasyc UMR CNRS 7253, Université de Technologie de Compiègne, Compiègne, France \\ ${ }^{2}$ Laboratoire de Méthodes de Conception de Systèmes, École nationale Supérieure d'Informatique, Algiers, Algeria \\ Email: \{mohamed - ali.kandi, hicham.lakhlef, madjid.bouabdallah, yacine.challal\}@hds.utc.fr
}

\begin{abstract}
Internet of Things (IoT) is a network made up of a large number of devices which are able to automatically communicate to computer systems, people and each other providing various services for the benefit of society. One of the main challenges facing the IoT is how to secure communication between these devices. Among all the issues, the Group Key Management is one of the most difficult. Although different approaches have been proposed to solve it, most of them use the same security parameters to secure all communications. Thus, if several services are provided by the network, communications within a service will be accessible to all network members even those which did not subscribe to it. Moreover, the compromise of a member will jeopardize all services. In this paper, we propose a highly scalable Multi-Group Key Management protocol for IoT which ensures the forward and backward secrecy, efficiently recovers from collusion attacks and guarantees the secure coexistence of several services in a single network. To achieve this, our protocol manages several groups with independent security parameters.

Index Terms-Internet of things, service, security, Group Key Management, forward and backward secrecy, collusion attack.
\end{abstract}

\section{INTRODUCTION}

The number of devices connected to Internet is constantly increasing since its appearance. Now that this number far exceeds that of people in the world, we are no longer talking about Internet but about Internet of Things. This emerging technology gives rise to revolutionary applications such as health care, environment monitoring, smart homes, smart cities...etc. The IoT devices, commonly called smart objects, are able to automatically communicate to computer systems, people and each other. The aim is to provide various services for the benefit of society. One of the main challenges facing the IoT is how to secure communication between these objects.

The Group Key Management $(G K M)$ is the core of secure communication. Its main role is to establish secure links between the members of a group. To achieve this, the GKM provides them with a secret cryptographic key that is used to encrypt the data exchanged [19]. Nevertheless, when a member leaves the group, it must no longer be able to decipher the future communications (forward secrecy). Also, if a node joins the group, it must not be able to decipher the previous ones (backward secrecy). Backward and forward secrecy are usually guaranteed by rekeying. Thus, when a node joins or leaves the group, the secret key is revoked and a new one is distributed to the remaining members. However, multiple compromised nodes can cooperate to regain access to the secret key. Such an attack is referred to as collusion attack [16].
The $G K M$ is a difficult issue especially for networks of constrained devices. Although different approaches have been proposed to solve it, most of them use the same parameters to secure all communications. Thus, if several services are provided by the network, communications within a service will be accessible to all network members even those which did not subscribe to it. Moreover, the compromise of a member will jeopardize all services. To address this problem, the hierarchical group access control has been proposed in [14] and [20]. However, this scheme cannot achieve a high performance when no hierarchy exists among services. A solution based on a Master Key Encryption has then been introduced in [9]. Nonetheless, using an asymmetric approach, this protocol is not well suited for networks of highly constraint devices. In this paper, we propose a highly scalable Multi-Group Key Management $(M G K M)$ protocol for IoT which ensures the forward and backward secrecy, efficiently recovers from collusion attacks and guarantees the secure coexistence of several services in the network. To achieve this, our protocol manages several groups with independent security parameters.

The remainder of this paper is organized as follows: related works are discussed in Section II. We detail then our solution in Section III. Section IV presents the security analysis of our protocol. In section $\mathrm{V}$, we evaluate the performance of our solution. Finally, we conclude in Section VI.

\section{RELATED WORKS}

According to the encryption technique used, the GKM schemes can be classified into three categories: symmetric, asymmetric and hybrid [19]. Symmetric approaches involve the use of the same key for encryption and decryption, while asymmetric ones use two different keys

Generally, symmetric schemes require less computation time and are more suitable for limited resources devices [18]. However, most of them suffer from high communication and memory overhead, are not scalable and are not resilient against compromise [11]. Symmetric approaches are usually based on Logical Key Hierarchy (LKH) [5, 13], Exclusion Basis Systems (EBS) [4, 7], polynomials [6], matrices [17]...etc.

On the other hand, asymmetric protocols are more secure and scalable. However, they usually require intensive computing, which makes them impractical on constrained devices. Despite this, some asymmetric schemes was proposed even for wireless sensor networks. Most of them implemented Elliptic 
Curve Cryptography (ECC) [1, 10, 12], CertificateLess Public Key Cryptography (CL-PKC) [8, 11], ID-Based Encryption (IBE) [3]...etc. Some works [2] proposed then hybrid schemes that combine both techniques (symmetric and asymmetric) to take advantages of each and overcome its disadvantages.

Regardless of the type of encryption used, only few researches considered the possibility of coexistence of several services in a single network. The authors in [14] and [20] proposed $M G K M$ schemes that achieve hierarchical group access control. However, the protocols cannot achieve a high performance when no hierarchy exists among services. The authors in [9] proposed then a new scheme called the Master Key Encryption Based $M G K M$. Nevertheless, the protocol is based on an asymmetric approach and is therefore not well suited for networks of highly constraint devices.

The symmetric protocol GREP [16] exploits the history of node joining to establish a total ordering among nodes. The aim is to make the rekeying process scalable and efficient. However, although nodes are organized into subgroups, their security parameters are not independent. All subgroups must then be rekeyed when a node gets compromised. On this basis, we propose a highly scalable $M G K M$ protocol for IoT which ensures the forward and backward secrecy, efficiently recovers from collusion attacks and guarantees the secure coexistence of several services in a single network.

\section{OUR SOLUTION}

The $M G K M$ we propose uses two layers. The upper layer manages multiple groups and assigns nodes to them according to the services to which they subscribe. On the other hand, the lower layer distributes the nodes of each group into logical subgroups in order to reduce the protocol overheads on them. The network is then divided into several groups, each of which is also partitioned into several subgroups (Figure 1). By doing this, the security parameters of services will be independent and the protocol is lighter for the network nodes.

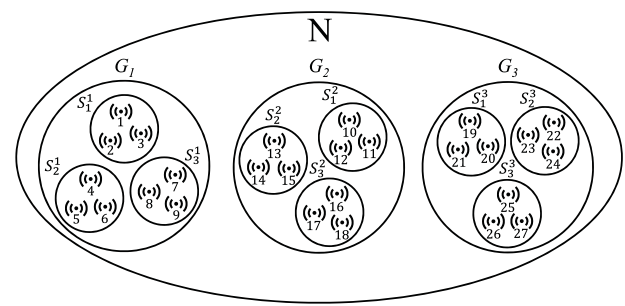

Fig. 1: Network partitioning according to services.

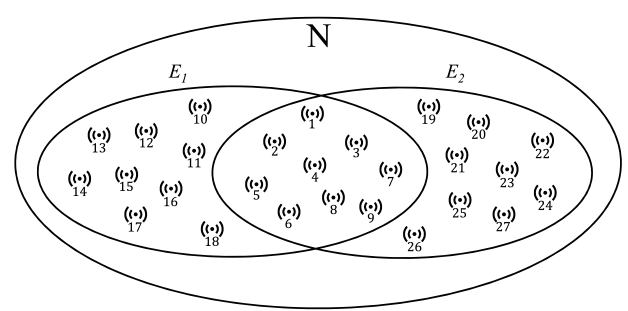

Fig. 2: Network partitioning achieved by the protocol.

\section{A. Services and groups management}

An IoT service is a transaction between two entities: a provider and a consumer. The former measures the state of the latter or initiates actions which will cause a change to it [15]. The provider is usually an object while the consumer can be a human, the environment or an other smart device. The main role of the $G K M$ is to establish secure communications between the objects. A smart device can then participate to a service as a provider, a consumer or both. It may also participate to different services, at the same time, and subscribe or unsubscribe from services at any time. The IoT can then be seen as a set of overlapping classes each gathering nodes which collaborate to provide a service and others that benefit from it (Figure 2). As these classes are overlapping, a group of the protocol cannot be associated to a service. Indeed, the independence of the group security parameters will then lose its meaning and the compromise of a node can jeopardize several groups. We propose then the creation of a group for each possible combination of services. A combination $A_{i}$ of $k_{i}$ services, of a finite set $E$ of $e$ services, is a subset of $k_{i}$ elements of $E$. The number of combinations, $n c$, is equal to:

$$
n c=\sum_{k=1}^{e} C_{e}^{k}=2^{e}-1
$$

The network $N$ is then partitioned into groups. Each group $G_{i}$ is associated with an $I D, g_{i d_{G_{i}}}$, which is unique within $N$. It contains then the nodes participating in the services of the combination $A_{i}$ associated to it. When an actual member subscribes or unsubscribes from services, it migrates from a group to another according to its new combination of services. The number of groups can reach $n c$ (Formula 1) if there are nodes participating in every possible combination of services. On the other hand, it cannot exceed the number of network nodes, $n$, because empty groups are not allowed. The maximum number of groups, $\max _{g}$, is therefore equal to:

$$
\max _{g}=\operatorname{Min}\left(2^{e}-1, n\right)
$$

Groups are created and removed as and when required and the probability of having only one node in each group is low. Their number can then be much smaller than $\max _{g}$. In Figure 2, two services $E_{1}$ and $E_{2}$ coexist in $N$. Three combinations are then possible: $A_{1}=\left\{E_{1}, E_{2}\right\}, A_{2}=\left\{E_{1}\right\}$ and $A_{3}=\left\{E_{2}\right\}$. Each group $G_{i}$ contains the nodes participating in the combination of services $A_{i}$ associated to it (Figure 1).

\section{B. Subgroups management}

To reduce the protocol costs for nodes, each group $G_{i}$ is in turn partitioned into a set of logical subgroups. A subgroup $S_{j}^{i}$ is associated with an $I D, \operatorname{sid}_{S_{j}^{i}}$, which is unique within $G_{i}$ and reflects its subgroups' total order. Given two subgroups $S_{j_{1}}^{i}$ and $S_{j_{2}}^{i}, \operatorname{sid}_{S_{j_{1}}^{i}}<\operatorname{sid}_{S_{j_{2}}^{i}}$ if and only if $S_{j_{1}}^{i}$ was created before $S_{j_{2}}^{i}$. Thereby, $S_{j_{1}}^{i}$ is considered as an elder kindred of $S_{j_{2}}^{i}$ whereas the latter is seen as a junior kindred of the former. Each subgroup $S_{j}^{i}$ is also associated to two subgroup tokens: a forward, $s t_{S_{j}^{i}}^{F}$, and a backward one, $s t_{S_{j}^{i}}^{B}$. In Figure 1, each group is partitioned into three subgroups. 


\section{Nodes management}

Each node $u$ is first assigned to a subgroup $S_{j}^{i}$ of the group $G_{i}$. The group is chosen according to the combination of services $A_{i}$ in which $u$ participates. On the other hand, the problem of the choice of the subgroup is treated by us in an ongoing work. The node $u$ is then associated with an $I D$, $n i d_{u}$, which is unique within $S_{j}^{i}$ and reflects, its members' total order. Given two nodes $u$ and $v, n i d_{u}<n i d_{v}$ if and only if $u$ has joined $S_{j}^{i}$ before $v$. Thus, $u$ is considered as an elder cognate of $v$ whereas $v$ is seen as a junior cognate of $u$.

Also, $u$ is associated to two node tokens: a forward, $t_{u}^{F}$, and a backward one, $t_{u}^{B}$. The node does not know neither its tokens $\left(t_{u}^{F}\right.$ and $\left.t_{u}^{B}\right)$ nor those of $S_{j}^{i}\left(s t_{S_{j}^{i}}^{F}\right.$ and $\left.s t_{S_{j}^{i}}^{B}\right)$. However, it stores the forward and backward node tokens associated to its elder and junior cognates, respectively, and the forward and backward subgroup tokens of the elder and junior kindred of $S_{j}^{i}$, respectively. Moreover, $u$ holds a secret node key, $K_{u}$, and shares a subgroup key, $K_{S^{i}}$, with its cognates and a group key, $K_{G_{i}}$, with all the nodes of $G_{i}$. Finally, $u$ stores a service key, $K_{E_{k}}$, for each service $E_{k}$ in which it participates. Note that unlike other keys, those of services do not intervene in the rekeying process and are only used to encrypt communication. The table in Figure 3 shows the keys and tokens held by the nodes of the group $G_{1}$ of the example presented in Figure 1.

\begin{tabular}{|c|c|c|c|c|c|c|c|c|}
\hline $\begin{array}{c}\text { Node } \\
\text { ID }\end{array}$ & $\begin{array}{c}\text { Node } \\
\text { key }\end{array}$ & $\begin{array}{c}\text { Subgroup } \\
\text { key }\end{array}$ & $\begin{array}{c}\text { Service } \\
\text { kevs }\end{array}$ & $\begin{array}{c}\text { Group } \\
\text { key }\end{array}$ & \multicolumn{2}{|c|}{ Node tokens } & \multicolumn{2}{|c|}{ Subgroup tokens } \\
\hline 1 & $K_{1}$ & \multirow{3}{*}{$K_{s_{1}^{1}}$} & \multirow{9}{*}{$K_{E_{1}}, K_{E_{2}}$} & \multirow{9}{*}{$K_{G_{1}}$} & & $t_{2}^{F}, t_{3}^{F}$ & \multirow{3}{*}{ - } & \multirow{3}{*}{$s t_{s_{2}^{1}}^{F}, s t_{s_{3}^{1}}^{F}$} \\
\hline 2 & $K_{2}$ & & & & $t_{1}^{B}$ & $t_{3}^{F}$ & & \\
\hline 3 & $K_{3}$ & & & & $t_{1}^{B}, t_{2}^{B}$ & & & \\
\hline 4 & $K_{4}$ & \multirow{3}{*}{$K_{S_{2}^{1}}$} & & & & $t_{5}^{F}, t_{6}^{F}$ & \multirow{3}{*}{$s t_{s_{1}^{1}}^{B}$} & \multirow{3}{*}{$s t_{S_{3}^{1}}^{F}$} \\
\hline 5 & $K_{5}$ & & & & $t_{4}^{B}$ & $t_{6}^{F}$ & & \\
\hline 6 & $K_{6}$ & & & & $t_{4}^{B}, t_{5}^{B}$ & - & & \\
\hline 7 & $K_{7}$ & \multirow{3}{*}{$K_{S_{3}^{1}}$} & & & - & $t_{8}^{F}, t_{9}^{F}$ & \multirow{3}{*}{$s t_{s_{1}^{1}}^{B}, s t_{s_{2}^{1}}^{B}$} & \multirow{3}{*}{ - } \\
\hline 8 & $K_{8}$ & & & & $t_{7}^{B}$ & $t_{9}^{F}$ & & \\
\hline 9 & $K_{9}$ & & & & $t_{7}^{B}, t_{8}^{B}$ & - & & \\
\hline
\end{tabular}

Fig. 3: Example of keys and tokens held by nodes.

In the following, we use the notations $t_{M}, K_{R}, K E K, H$ and $K D F$ to refer to a master node token, a refresh key, a key encryption key, a one-way hash function and a pseudo-random key derivation function, respectively. Also, $\{m\} K$ means the message $m$ is encrypted using $K$ and $M G K M \rightarrow R:<m>$ denotes that the $M G K M$ sends $<m>$ to the node(s) $R$.

1) Rekeying upon joining: When a node $u$ joins the network, the $M G K M$ starts by assigning it to a given subgroup $S_{j}^{i}$ of the group $G_{i}$. Due to space constraints, we assume that the subgroup $S_{j}^{i}$ already exists. It determines then $n i d_{u}, K_{u}$ and $t_{u}^{B}$. To ensure the backward secrecy, the $M G K M$ randomly generates $K_{R}$ and $t_{M}$. Then, using the $K D F$, it computes $t_{u}^{F}$ and the new group and subgroup keys, $K_{G_{i}}^{+}$and $K_{S^{i}}^{+}$(Formulas 3 to 5). The $K D F$ is also used to update the key $K_{E_{k}}$ of each service $E_{k}$ to which $u$ subscribes (Formula 6). Finally, the $M G K M$ discards $t_{M}$ and $K_{R}$ and broadcasts the messages $J M 1$ to JM3. The message JM1 is sent to the nodes of $S_{j}^{i}$ and is encrypted using its actual key $K_{S_{j}^{i}}$. The message JM2 is intended to those belonging to the other subgroups of $G_{i}$ and is then encrypted using its actual key $K_{G_{i}}$. For each group $G_{s}\left(G_{s} \neq G_{i}\right)$, which shares at least a service with $G_{i}$ $\left(A_{s} \cap A_{i} \neq \emptyset\right)$, the message $J M 3$ is sent encrypted using $K_{G_{s}}$.

$$
\begin{aligned}
& t_{u}^{F}=K D F\left(t_{M} \| K_{R}\right) \quad \text { (3) } \quad K_{G_{i}}^{+}=K D F\left(K_{G_{i}} \| K_{R}\right) \\
& K_{S_{j}^{i}}^{+}=K D F\left(K_{S_{j}^{i}} \| K_{R}\right) \quad \text { (5) } \quad K_{E_{k}}^{+}=K D F\left(K_{E_{k}} \| K_{R}\right) \\
& J M 1: M G K M \rightarrow S_{j}^{i}:<\operatorname{nid}_{u},\left\{t_{M}, K_{R}\right\} K_{S_{j}^{i}}> \\
& \text { JM2 :MGKM } \rightarrow G_{i}:<\left\{K_{R}\right\} K_{G_{i}}> \\
& \text { JM3 : MGKM } \rightarrow G_{s}:<\left\{A_{s} \cap A_{i}, K_{R}\right\} K_{G_{s}}>
\end{aligned}
$$

Upon receiving $J M 1$, a node in $S_{j}^{i}$ uses $K_{S_{j}^{i}}$ to retrieve $t_{M}$ and $K_{R}$, computes $t_{u}^{F}, K_{G_{i}}^{+}$and $K_{S_{j}^{i}}^{+}$and updates its service keys (Formulas 3 to 6). Upon receiving JM2, a node in $S^{i}$ $\left(S^{i} \in G_{i}\right.$ and $S^{i} \neq S_{j}^{i}$ ) uses $K_{G_{i}}$ to retrieve $K_{R}$, computes $K_{G_{i}}^{+}$and updates its service keys (Formulas 4 and 6). Upon receiving $J M 3$, a node in $G_{s}$ uses $K_{G_{s}}$ to retrieve $K_{R}$ and updates the shared service keys (Formula 6). Finally, the $M G K M$ provides $u$, via a secure channel, with $K_{u}, K_{G_{i}}^{+}, K_{S_{i}^{i}}^{+}$, the service keys, the backward tokens of its cognates and the backward and forward ones of the kindreds of $S_{j}^{i}$.

2) Rekeying upon leaving: When a node $u$ leaves a subgroup $S_{j}^{i}$ of the group $G_{i}$, the cryptographic material it holds get compromised and must be revoked. By construction, four tokens remain secret $\left(t_{u}^{F}, t_{u}^{B}, s t_{S_{j}^{i}}^{F}\right.$ and $\left.s t_{S_{j}^{i}}^{B}\right)$ and are then used to rekey the network. Due to space constraints, we do not consider the case when $S_{j}^{i}$ becomes empty. Thus, to ensure the forward secrecy, the $M G K M$ starts by generating $K_{R}$. Then, using the $K D F$, it computes $K_{G_{i}}^{+}, K_{S_{j}^{i}}^{+}$, four $K E K s$ (Formulas 4, 5 and 7 to 10) and updates the service keys $u$ held (Formula 6). Next, the $M G K M$ removes the node key and tokens of $u$ and uses $K_{R}$ and $H$ to update the tokens it knows (Formulas 11 and 12). Finally, the $M G K M$ broadcasts the messages $L M 1$ to $L M 3$ and discards $K_{R}$ and the $K E K s$. The message $L M 1$ is sent to the nodes of $S_{j}^{i}$ and is encrypted using the $K E K s$ generated from the tokens of $u$ ( $K_{F}$ and $K_{B}$ ). The message $L M 2$ is intended to those belonging to the other subgroups of $G_{i}$ and is then encrypted using the $K E K s$ generated from the tokens of $S_{j}^{i}\left(K_{F}^{S}\right.$ and $\left.K_{B}^{S}\right)$. For each group $G_{s}\left(G_{s} \neq G_{i}\right)$ which shares at least a service with $G_{i}\left(A_{s} \cap A_{i} \neq \emptyset\right), L M 3$ is sent encrypted by means of $K_{G_{s}}$.

$$
\begin{aligned}
& K_{F}=K D F\left(t_{u}^{F}\right) \quad \text { (7) } \quad K_{B}=K D F\left(t_{u}^{B}\right) \\
& K_{F}^{S}=K D F\left(s t_{S_{j}^{i}}^{F}\right) \quad \text { (9) } \quad K_{B}^{S}=K D F\left(s t_{S_{j}^{i}}^{B}\right) \\
& t^{+} \leftarrow H\left(t \| K_{R}\right) \quad(11) \quad s t^{+} \leftarrow H\left(s t|| K_{R}\right) \\
& L M 1: M G K M \rightarrow S_{j}^{i}:<n i d_{u},\left\{K_{R}\right\} K_{F},\left\{K_{R}\right\} K_{B}> \\
& \text { LM2 }: M G K M \rightarrow G_{i}:<\operatorname{sid}_{S_{j}^{i}},\left\{K_{R}\right\} K_{F}^{S},\left\{K_{R}\right\} K_{B}^{S}> \\
& L M 3: M G K M \rightarrow G_{s}:<\left\{A_{s} \cap A_{i}, K_{R}\right\} K_{G_{s}}>
\end{aligned}
$$

Upon receiving $L M 1$, a node $v$ in $S_{j}^{i}(v \neq u)$ computes either $K_{F}$, if $n i d_{v}<n i d_{u}$, or $K_{B}$ otherwise (Formulas 7 or 8) and retrieves $K_{R}$. Then, it removes either $t_{u}^{B}$ or $t_{u}^{F}$, computes $K_{G_{i}}^{+}$and $K_{S_{i}^{i}}^{+}$and updates the service keys and tokens $u$ knows (Formulas 4 to 6, 11 and 12). Upon receiving $L M^{2}$, a node in $S^{i}\left(S^{i} \in G_{i}\right.$ and $\left.S^{i} \neq S_{j}^{i}\right)$ computes either $K_{F}^{S}$, if $\operatorname{sid}_{S^{i}}<s i d_{S_{j}^{i}}$, or $K_{B}^{S}$ otherwise (Formula 9 or 10) and retrieves $K_{R}$. Then, it computes $K_{G_{i}}^{+}$and updates the service keys and the subgroup tokens $u$ knows (Formulas 4, 6 and 12). Upon receiving $L M 3$, a node in $G_{s}$ uses $K_{G_{s}}$ to retrieve $K_{R}$ and updates the shared service keys (Formula 6). 
3) Recovering from collusion attack: In case of collusion attack, multiple compromised nodes share their information to regain access to the group and service keys. A subgroup, a group or a service is compromised if it contains at least one compromised node. To recover from it, the MGKM starts by generating $K_{R}$, removing the tokens of the evicted nodes and updating those they know (Formulas 11 and 12). Then, it computes a new key for each compromised group or service (Formulas 4 and 6). Regarding subgroups, three cases arise.

a) Rekeying a compromised subgroup $S_{j}^{i}$ : The $M G K M$ determines first its eldest $\left(u_{e}\right)$ and youngest $\left(u_{y}\right)$ compromised members. By construction, the tokens $t_{u_{e}}^{F}$ and $t_{u_{y}}^{B}$ remain secret for the evicted nodes and are used to generate two $K E K s$ (Formulas 7 and 8). The $M G K M$ utilizes then these keys to encrypt the message $R M 1$ and broadcasts it. However, non evicted nodes, that are junior cognates of $u_{e}$ and elder cognates of $u_{y}$, hold only compromised tokens. Thus, for each of them, the $M G K M$ sends the unicast message RM2 encrypted by the node key. If a non compromised node in $S_{j}^{i}$ receives $R M 1$, it calculates one of the KEKs and uses it to retrieve $K_{R}$. On the other hand, if the node receives $R M 2$, it directly uses its secret key to retrieve $K_{R}$. In both cases, the node utilizes $K_{R}$ to compute $K_{G_{i}}^{+}$as well as $K_{S_{i}^{i}}^{+}$and to update the service keys it knows (Formulas 4 to 6). Finally, it removes the tokens of its evicted cognates and updates those they held (Formula 11).

b) Rekeying non compromised subgroups of a compromised group $G_{i}$ : The $M G K M$ determines first the eldest $\left(S_{e}^{i}\right)$ and youngest $\left(S_{y}^{i}\right)$ compromised subgroups of $G_{i}$. By construction, the tokens $s t_{S_{e}^{i}}^{F}$ and $s t_{S_{y}^{i}}^{B}$ remain secret for the evicted nodes and are used to generate two KEKs (Formulas 9 and 10). The $M G K M$ utilizes then these keys to encrypt the message $R M 3$ and broadcasts it. However, nodes of the non compromised subgroups, which are junior kindreds of $S_{e}^{i}$ and elder kindreds of $S_{y}^{i}$, hold only compromised subgroup tokens. Thus, for each of them, the $M G K M$ broadcasts $R M_{4}$ encrypted by means of the subgroup key. If a node of a non compromised subgroup receives $R M 3$, it calculates one of the $K E K s$ and uses it to retrieve $K_{R}$. Otherwise, if the node receives $R M_{4}$, it directly uses its subgroup key to retrieve $K_{R}$. In both cases, the node utilizes $K_{R}$ to compute $K_{G_{i}}^{+}$and update the service keys it knows (Formulas 4 and 6). Finally, it updates all the compromised subgroup tokens (Formula 12).

c) Rekeying non compromised subgroups with compromised services: Although a subgroup belongs to a non compromised group $G_{s}$, its members can share some services with the compromised ones. In this case, the $M G K M$ broadcasts the message $R M 5$ encrypted by means of the group key, $K_{G_{s}}$. Upon receiving the message, a node in $G_{s}$ retrieves $K_{R}$ and updates its compromised service keys.

$$
\begin{gathered}
\text { RM1 }: M G K M \rightarrow S_{j}^{i}:<\text { uid }_{u_{e}}, \text { uid }_{u_{y}},\left\{K_{R}\right\} K_{F},\left\{K_{R}\right\} K_{B}> \\
\text { RM2 }: M G K M \rightarrow u:<\operatorname{uid}_{u_{e}}, u i d_{u_{y}},\left\{K_{R}\right\} K_{u}> \\
\text { RM3 }: M G K M \rightarrow G_{i}:<\operatorname{sid}_{S_{e}^{i}}, \operatorname{sid}_{S_{y}^{i}},\left\{K_{R}\right\} K_{F}^{S},\left\{K_{R}\right\} K_{B}^{S}> \\
\text { RM4 }: M G K M \rightarrow S^{i}:<\operatorname{sid}_{S_{e}^{i}}, \operatorname{sid}_{S_{y}^{i}},\left\{K_{R}\right\} K_{S^{i}}> \\
\text { RM5 }: M G K M \rightarrow G_{s}:<\left\{K_{R}\right\} K_{G_{s}}>
\end{gathered}
$$

4) Rekying upon group changing: When an actual member $u$ subscribes or unsubscribes from services, it is moved from a group $G_{p}$ to another $G_{n}$. The choice of $G_{n}$ depends on the new combination of services $A_{n}$ in which $u$ participates.

As $u$ leaves the subgroup $S_{j}^{p}$ of $G_{p}$, the cryptographic material it holds needs to be changed. By construction, the tokens $t_{u}^{F}, t_{u}^{B}, s t_{S_{j}^{p}}^{F}$ and $s t_{S_{i}^{p}}^{B}$ remain secret for $u$ and are used to rekey $G_{p}$. To ensure the forward secrecy, the $M G K M$ generates $K_{R}$, uses the $K D F$ to compute $K_{G_{p}}^{+}, K_{S_{j}^{p}}^{+}$as well as four KEKs (Formulas 4, 5 and 7 to 10) and updates the tokens $u$ knows (Formulas 11 and 12). Also, as $u$ joins the subgroup $S_{j}^{n}$ of $G_{n}$, the $M G K M$ determines its new $I D$ and backward token. To ensure the backward secrecy, the $M G K M$ uses the $K D F$ to generate a new $t_{u}^{F}$, to compute $K_{G_{n}}^{+}$and $K_{S_{j}^{n}}^{+}$ (Formulas 3 to 5 ) and to update the key of each service $E_{k}$ to which $u$ subscribes or unsubscribes (Formula 6). Finally, the MGKM broadcasts the messages EM1 to EM5.

The message $E M 1$ is sent to the nodes of $S_{j}^{p}$ and is encrypted using the $K E K s$ generated from the tokens of $u$. On the other hand, EM2 is intended to those of the other subgroups of $G_{p}$ and is encrypted using the $K E K s$ generated from the tokens of $S_{j}^{p}$. EM3 is sent to the nodes of $S_{j}^{n}$ and $E M_{4}$ to those of the other subgroups of $G_{n}$. They are then encrypted using $K_{S_{j}^{n}}$ and $K_{G_{n}}$, respectively. For each group $G_{s}\left(G_{s} \neq G_{p}\right.$ and $\left.G_{s} \neq G_{n}\right)$ participating in a service $E_{k}$, the $M G K M$ broadcasts the message EM5 encrypted using $K_{G_{s}}$. Finally, a last message is used to provide $u$ with its new cryptographic material and is encrypted by means of $K_{u}$.

$$
\begin{gathered}
\text { EM1 }: M G K M \rightarrow S_{j}^{p}:<\operatorname{nid}_{u},\left\{K_{R}\right\} K_{F},\left\{K_{R}\right\} K_{B}> \\
\text { EM2 }: M G K M \rightarrow G_{p}:<\operatorname{sid}_{S_{j}^{p}},\left\{K_{R}\right\} K_{F}^{S},\left\{K_{R}\right\} K_{B}^{S}> \\
\text { EM3 }: M G K M \rightarrow S_{j}^{n}:<n i d_{u},\left\{t_{M}, K_{R}\right\} K_{S_{j}^{n}}> \\
\text { EM4 }: M G K M \rightarrow G_{n}:<\left\{K_{R}\right\} K_{G_{n}}> \\
\text { EM5 }: M G K M \rightarrow G_{s}:<\left\{L E_{s}, K_{R}\right\} K_{G_{s}}>
\end{gathered}
$$

Upon receiving $E M 1$, a node $v$ in $S_{j}^{p}(v \neq u)$ computes either $K_{F}$, if $n i d_{v}<n i d_{u}$, or $K_{B}$ otherwise (Formula 7 or 8 ) and retrieves $K_{R}$. Then, it computes $K_{G_{p}}^{+}$and $K_{S_{j}^{p}}^{+}$(Formulas 4 and 5), updates the tokens $u$ knows (Formulas 11 and 12) and removes either $t_{u}^{B}$ or $t_{u}^{F}$. Moreover, $v$ updates the keys of services from which $u$ unsubscribed (Formula 6). Upon receiving $L M 2$, a node in $S^{p}\left(S^{p} \in G_{p}\right.$ and $\left.S^{p} \neq S_{j}^{p}\right)$ computes either $K_{F}^{S}$, if $\operatorname{sid}_{S^{p}}<s i d_{S_{j}^{p}}$, or $K_{B}^{S}$ otherwise (Formula 9 or 10) and retrieves $K_{R}$. Then, it computes $K_{G_{n}}^{+}$and updates the subgroup tokens $u$ knows (Formulas 4 and 12). Also, the node updates the keys of services from which $u$ unsubscribed (Formula 6). Upon receiving $E M 3$, a node in $S_{j}^{n}$ uses $K_{S_{j}^{n}}$ to retrieve $t_{M}$ and $K_{R}$ and computes $t_{u}^{F}, K_{G_{n}}^{+}$and $K_{S_{j}^{n}}^{+}$(Formulas 3 to 5). Also, the node updates the keys of services to which $u$ subscribed (Formula 6). Upon receiving EM/4, a node in $S^{n}$ $\left(S^{n} \in G_{n}\right.$ and $S^{n} \neq S_{j}^{n}$ ) uses $K_{G_{n}}$ to retrieve $K_{R}$, computes $K_{G_{n}}^{+}$(Formula 4) and updates the keys of services to which $u$ subscribed (Formula 6). Finally, upon receiving JM5, a node in $G_{s}$ uses $K_{G_{s}}$ to retrieve $K_{R}$ and the list of the shared services, $L E_{s}$, and updates their keys (Formula 6). 


\section{SECURITy ANALYSis}

Before a node $u$ joins a subgroup $S_{j}^{i}$ of a group $G_{i}$, the $M G K M$ rekeys both of them as well as all services involved. To achieve this, the $M G K M$ uses the messages JM1 to JM3 (if $u$ joins the network) or EM3 to EM5 (when it changes group). The messages JM1 and EM3 allow the members of $S_{j}^{i}$ to calculate the new subgroup, group and service keys. They are encrypted using the actual subgroup key, $K_{S_{j}^{i}}$. On the other hand, JM2 and EM4 enable the other nodes of $G_{i}$ to calculate the new group and service keys. They are then encrypted using the actual group key, $K_{G_{i}}$. Finally, JM3 and EM5 allow the members of the other groups which participate to the same services as $u$ to calculate the new service keys. They are encrypted by means of their group keys. At the end of the process of rekeying, the $M G K M$ provides $u$ with the new keys, via a secure channel (if $u$ joins the network) or using a message encrypted by means of $K_{u}$ (if $u$ changes group). The network is rekeyed and $u$ gets the new keys without access to the old ones. The backward secrecy is then guaranteed.

After a node $u$ leaves a subgroup $S_{j}^{i}$ of a group $G_{i}$, the $M G K M$ rekeys both of them as well as all the services involved. To achieve this, the $M G K M$ uses the messages $L M 1$ to $L M 3$ (if $u$ has left the network), the messages EM1, EM2 and EM5 (when it changes group) and the messages $R M 1$ to RM5 (in case of collusion attack). The messages $L M 1$, $E M 1, R M 1$ and $R M 2$ allow the members of $S_{j}^{i}$ to calculate the new subgroup, group and service keys. They are encrypted using their node keys or the $K E K s$ generated from the tokens of $u$. On the other hand, LM2, EM2, RM3 and RM4 enable the other nodes of $G_{i}$ to calculate the new group and service keys. They are then encrypted using their subgroup keys or the $K E K s$ generated from the tokens of $S_{j}^{i}$. Finally, LM3, EM5 and RM5 allow the members of the other groups which participate to the same services as $u$ to calculate the new service keys. They are encrypted by means of their group keys. By construction, $u$ does not know any of the keys used to encrypt these messages and is then excluded from the process of rekeying. Since it cannot get access to the new keys or any future incarnation of them, the forward secrecy is guaranteed.

In our solution, the compromise of a node does not affect the services in which it does not participate. This is due, firstly, to the fact that the members of the same group participate to the same services and, secondly, because nodes belonging to different groups do not share any security parameter if they have no service in common. Let us consider two groups $G_{i}$ and $G_{j}$ associated to the combinations $A_{i}$ and $A_{j}$, respectively. When a node $u$ of the group $G_{i}$ gets compromised, only the service keys of $A_{i}$ are exposed. If $G_{i}$ and $G_{j}$ share some services $\left(A_{i} \cap A_{j} \neq \emptyset\right)$, the keys of services to which the members of $G_{j}$ participate but not those of $G_{i}\left(A_{i} \backslash A_{j}\right)$ remain secret. Indeed, $u$ does not know them. Furthermore, if the groups do not share services $\left(A_{i} \cap A_{j}=\emptyset\right)$, any of the service keys of $A_{j}$ gets compromised. In both cases, only the services in which $u$ participates are compromised. Thus, the compromise of a service has no effect on the others.

\section{PERFormance EVAluation}

In the following, we use the notations $m_{i}^{j}, p_{i}$ and $k_{i}$ to refer to the number of nodes in $S_{j}^{i}$ and that of subgroups and services in $G_{i}$. In a collusion attack, we consider that $c$ nodes are evicted. We assume that $K D F$ and $H$ require the same computing cost and that keys and tokens have the same size.

\section{A. Overheads on the MGKM}

In a network of $n$ nodes, the $M G K M$ stores $n$ node keys, $n$ subgroup keys, $\max _{g}$ group keys, $e$ service keys, 2.n node tokens and 2.n subgroup tokens, in the worst case. If we assume that $e \leq n$, the storage will be of the order of $O(n)$.

When a node $u$ joins the subgroup $S_{j}^{i}$ of $G_{i}$, the $M G K M$ generates $K_{R}, t_{M}, K_{u}, t_{u}^{F}, t_{u}^{B}$ and updates $K_{S_{j}^{i}}, K_{G_{i}}$ and $k_{i}$ service keys. It performs then $k_{i}+7$ hash function executions. It also encrypts and sends messages JM1, JM2 and, for each group sharing services with $G_{i}$, the message JM3. As the number of groups reaches $\max _{g}$, in the worst case, the $M G K M$ can encrypt and send up to $\max _{g}+1$ messages.

When $u$ leaves the network, the $M G K M$ generates $K_{R}$, $4 K E K s$ and updates $K_{S_{j}^{i}}, K_{G_{i}}, k_{i}$ service keys, $m_{j}^{i}-2$ node tokens and $p_{i}-1$ subgroup tokens. It performs then $p_{i}+m_{i}^{j}+k_{i}+4$ hash function executions. It also encrypts and sends the messages $L M 1, L M 2$ and, for each group which shares services with $G_{i}$, the message LM3. The MGKM encrypts and sends then $\max _{g}+1$ messages in the worst case.

When $u$ changes group, the $M G K M$ generates $K_{R}, t_{M}$ and $4 K E K s$, updates 2 group keys, 2 subgroup keys, $m_{j}^{i}-2$ node tokens, $p_{i}-1$ subgroup tokens and the $k_{u}$ service keys to which the node subscribes or unsubscribes. It performs then $p_{i}+m_{i}^{j}+k_{u}+7$ hash function executions. It also encrypts and sends the messages EM1 to EM4 and, for each group associated with a compromised service, the message EM5. The $M G K M$ encrypts and sends then $\max _{g}+3$ messages in the worst case.

The worst case for a recovery from collusion attack is when all nodes are rekeyed using the unicast message RM2 (all subgroups and all their node tokens are compromised). In this case, the $M G K M$ encrypts and sends $n-c$ messages. It also updates all subgroup, group and service keys. The communication and computing are then of the order of $O(n)$.

To sum up, the storage overheads for the $M G K M$ are of the order of $O(n)$. Also, the communication and computing costs are proportional to $\max _{g}$ (if a node joins, leaves or changes group) or of the order of $O(n)$ (in the case of collusion attack). Figure 4 illustrates the variation of $\max _{g}$ according to $n$ and $e$. However, in our analysis, we consider the worst case whose probability of occurring is low. The costs are much lighter in the general case. Moreover, this is usually not a problem in practice, since the $M G K M$ has plentiful of resources. It is on the side of nodes that the protocol must be light.

\section{B. Overheads on nodes}

A node $u$, belonging to a subgroup $S_{j}^{i}$ in $G_{i}$, stores $K_{u}$, $K_{S_{j}^{i}}, K_{G_{i}}, k_{i}$ service keys, $m_{i}^{j}-1$ node tokens and $p_{i}-1$ subgroup tokens. It holds then $p_{i}+m_{i}^{j}+k_{i}+1$ keys. 
The worst case for $u$ when a node $v$ joins $G_{i}$ ( $v$ joins the network or changes group) is that $v$ is assigned to $S_{j}^{i}$. The node receives then one message, decrypts two keys, generates $t_{v}^{F}$ and updates $K_{S_{i}^{i}}, K_{G_{i}}$ and $k_{i}$ service keys. It performs then 2 decryptions and $k_{i}+3$ hash function executions.

The worst case for $u$ when a node $v$ leaves $G_{i}$ ( $v$ leaves the network or changes group) is that they are cognates. The node $u$ receives then one message, decrypts one key, calculates one $K E K$ and updates $K_{S_{i}^{i}}, K_{G_{i}}, k_{i}$ service keys, $m_{i}^{j}-2$ node tokens and $p_{i}-1$ subgroup tokens. It performs then one decryption and $p_{i}+m_{i}^{j}+k_{i}$ hash function executions.

When a recovery from collusion attack is performed, the worst case for a node $u$ is that some of its cognates are evicted. The node receives then one message, decrypts one key and updates $K_{S_{i}^{i}}, K_{G_{i}}, k_{i}$ service keys, $m_{i}^{j}-c-1$ node tokens and $p_{i}-1$ subgroup tokens. The node performs then one decryption and $p_{i}+m_{i}^{j}+k_{i}-c$ hash function executions.

To sum up, the communication for nodes is constant $(O(1))$ and the storage and calculation are proportional to $p_{i}+m_{i}^{j}+k_{i}$. If the number of services is negligible when compared to the size of the network and if the $n$ nodes of the network are uniformly distributed (i.e. $\forall i, \forall j$, $p_{i} \simeq m_{i}^{j} \simeq \sqrt{n}$ ), the costs will be on average of the order of $O(\sqrt{n})$. Our solution is then efficient and scalable as, even if $n$ increases, the protocol is affordable for constrained nodes.

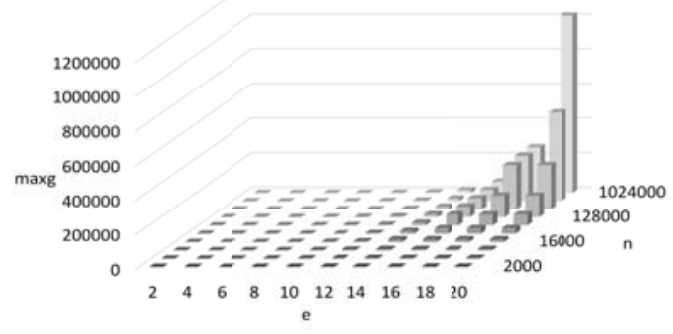

Fig. 4: Variation of $\max _{g}$ according to $n$ and $e$.

\section{Conclusion}

In this paper, we presented a highly scalable Multi-Group Key Management protocol for IoT which ensures the forward and backward security, efficiently recovers from collusion attacks and guarantees the secure coexistence of several services in the network. Our protocol manages several groups having independent security parameters. Each group is then associated to a combination of services. When a node joins the network, the protocol assigns it to the group associated to the combination of services to which it subscribes. When an actual member subscribes or unsubscribes from services, it migrates from a group to another according to the new combination of services. Our protocol efficiently rekeys groups, subgroups and services after a node joins or leaves the network, changes group or when a set of colluding nodes are evicted. In future works, we intend to decentralize the protocol. Cryptographic material will then be spread across more than one entity in order not to have a single point of failure and to make it more difficult to access or modify this secret material.

\section{ACKNOWLEDGMENTS}

This work was carried out and funded by Heudiasyc UMR CNRS 7253 and the Labex MS2T.

\section{REFERENCES}

[1] M. Alagheband and M.R. Aref. "Dynamic and secure key management model for hierarchical heterogeneous sensor networks". In: IET Information Security 6.4 (2012), pp. 271-280.

[2] R. Azarderakhsh, A. Reyhani-Masoleh and Z. Abid. "A key management scheme for cluster based wireless sensor networks". In: Embedded and Ubiquitous Computing, 2008. EUC'08. IEEE/IFIP Int. Conf. on. Vol. 2. IEEE. 2008, pp. 222-227.

[3] K. Chatterjee, A. De and D. Gupta. "An improved ID-Based key management scheme in wireless sensor network". In: Int. Conf. in Swarm Intelligence. Springer. 2012, pp. 351-359.

[4] C. Chen, Z. Huang, Q. Wen and Y. Fan. "A novel dynamic key management scheme for wireless sensor networks". In: Broadband Network and Multimedia Technology (IC-BNMT), 2011 4th IEEE International Conference on. IEEE. 2011, pp. 549-552.

[5] G. Dini and I.M. Savino. "S2rp: a secure and scalable rekeying protocol for wireless sensor networks". In: Mobile Adhoc and Sensor Systems, IEEE Int. Conf. on. 2006, pp. 457-466.

[6] A. Diop, Y. Qi and Q. Wang. "Efficient group key management using symmetric key and threshold cryptography for cluster based wireless sensor networks". In: International Journal of Computer Network and Information Security 6.8 (2014), p. 9.

[7] R. Divya and T. Thirumurugan. "A novel dynamic key management scheme based on hamming distance for wireless sensor networks". In: Computer, Communication and Electrical Technology (ICCCET), 2011 International Conference on. IEEE. 2011, pp. 181-185.

[8] D. Mall, K. Konaté and A.K. Pathan. "ECL-EKM: An enhanced Certificateless Effective Key Management protocol for dynamic WSN". In: Networking, Systems and Security (NSysS), 2017 International Conference on. IEEE. 2017, pp. 150-155.

[9] M. Park, Y. Park, H. Jeong and S. Seo. "Secure multiple multicast services in wireless networks". In: IEEE Transactions on Mobile Computing (2012).

[10] S.M.M Rahman and K. El-Khatib. "Private key agreement and secure communication for heterogeneous sensor networks". In: Journal of Parallel and Distributed Computing 70.8 (2010), pp. 858-870.

[11] S.H. Seo, J. Won, S. Sultana and E. Bertino. "Effective key management in dynamic wireless sensor networks". In: IEEE Transactions on Information Forensics and Security 10.2 (2015), pp. 371-383.

[12] S.R. Singh, A.K. Khan and T.S. Singh. "A New Key Management Scheme for Wireless Senm Networks using an Elliptic Curve". In: Indian Journal of Science and Technology 10.13 (2017).

[13] J. Son, J. Lee and S. Seo. "Topological key hierarchy for energyefficient group key management in wireless sensor networks". In: Wireless personal communications 52.2 (2010), p. 359.

[14] Y. Sun and K.R. Liu. "Hierarchical group access control for secure multicast communications". In: IEEE/ACM Transactions on Networking 15.6 (2007), pp. 1514-1526.

[15] M. Thoma, S. Meyer, K. Sperner, S. Meissner and T. Braun. "On iot-services: Survey, classification and enterprise integration". In: Green Computing and Communications (GreenCom), 2012 IEEE International Conference on. IEEE. 2012, pp. 257-260.

[16] M. Tiloca and G. Dini. "GREP: A group rekeying protocol based on member join history". In: Computers and Communication (ISCC) 2016 IEEE Symposium on. IEEE. 2016, pp. 326-333.

[17] I. Tsai, C. Yu, H. Yokota and S. Kuo. "Key Management in Internet of Things via Kronecker Product”. In: Dependable Computing, 2017 IEEE 22nd Pacific Rim International Symposium on. Pp. 118-124.

[18] F. Zhan, N. Yao, Z. Gao and G. Tan. "A novel key generation method for wireless sensor networks based on system of equations". In: Journal of Network and Computer Applications 82 (2017), pp. 114-127.

[19] J. Zhang and V. Varadharajan. "Wireless sensor network key management survey and taxonomy". In: Journal of Network and Computer Applications 33.2 (2010), pp. 63-75.

[20] Q. Zhang and Y. Wang. "A centralized key management scheme for hierarchical access control". In: Global Telecommunications Conference, 2004. GLOBECOM'04. IEEE. Vol. 4. 2004, pp. 2067-2071. 